\title{
EIN NEUARTIGES ORGAN-UNTERSTÜTZUNGS-SYSTEM ZUR LEBENDERHALTUNG VON ARTERIEN UNTER PHYSIOLOGISCHEN BEDINGUNGEN
}

\author{
M.W. Waliszewski, N. Stergiopulos, J.-J. Meister \\ Laboratoire de Génie Médical, PSE-Ecublens, EPFL, CH-1015 Lausanne, Schweiz
}

\section{EINLEITUNG}

Durch Arteriosklerose hervorgerufene HerzKreislauf-Erkrankungen sind die Haupttodesursache in industrialisierten Ländern. Aufgrund der Vielzahl der einflußnehmenden Größen ist die Kontrollierbarkeit der hämodynamischen Bedingungen in der Arteriosklerose-Forschung, wie Blutdruck und -strömung, von höchster Bedeutung. Während in Zellkulturen bei guter Kontrollierbarkeit nur ein Bruchteil des arteriellen Gewebes modelliert werden kann, mangelt es bei in-vivo Tierversuchen unter physiologischen Testbedingungen an dieser Kontrollierbarkeit. Die Anfang der 80iger Jahre von D.L. Fry in den USA entwickelte OrganUnterstützungs-Technologie wurde an der EPF Lausanne zu einem universellen Testsystem von Arterien weiterentwickelt. Mit diesem OrganUnterstützungs-System (OUS) können metabolisch unterstützte Arterien durch Simulation ihrer in-vivo Umgebung für Tage unter kontrollierten Testbedingungen am Leben erhalten werden. Ziel des laufenden Projektes ist es das sog. "Wall Remodeling" von Arterien unter kontrollierten, hypertonen Testbedingungen zu untersuchen.

\section{METHODE}

Das an der EPF Lausanne entwickelte OrganUnterstützung-System besteht aus dem Doppelkammer Arterien-Simulations-system, einem Pentium $^{\mathrm{TM}} \mathrm{PC}$, einem Ultraschall-Meßsystem und $\operatorname{der} \mathrm{CO}_{2} / \mathrm{O}_{2}$ Gasversorgung. Sämtliche Datenerfassungsroutinen, MeB- und Regelkreise wurden in einem eigenentwickelten Software Paket $\left(C_{++}\right)$ realisiert, die sog. Protokolldateien von Temperatur, pH-Wert und Druck des Perfusionsmediums sowie $\mathrm{CO}_{2}$ - und $\mathrm{O}_{2}$ - Gehalt der OUS-Atmosphäre erstellt.

Die Funktion der Lungen wird von einem $\mathrm{CO}_{2} / \mathrm{O}_{2}$ Gasgemisch simuliert, wohingegen die Nierenfunktion ggf. durch ein Dialysemodul ersetzt werden kann.

Zur Arterienpräparation wird die A. communis sinistra mit dem truncus bicaroticus von Kälbern entnommen. Nach anschließendem Sezieren werden zwei etwa $25 \mathrm{~mm}$ lange Teilstücke von überflüssigem Gewebe entfernt, bevor sie an speziellen Konnektoren angebracht werden. Diese Konnektor-Arterien-Anordnungen werden mit ihrer Adventitia-Umströmungskammer in das OUS eingesetzt. Als Perfusionsmedium wird gepooltes Kälberserum (Biochrom KG, Berlin) benutzt, welches durch Zugabe von $\mathrm{NaHCO}_{3}$ auf eine standardisiert Konzentration von $27 \mathrm{mmol} / \mathrm{l}$ gebracht wird. Als Umströmungsmedium wird eine Tyrodelösung mit der gleichen Bikarbonatkonzentration verwendet. Die Vitalität des Gewebes wurde anhand von Nukleotid Live/Dead ${ }^{\mathrm{TM}}$ Stains (Molecular Probes, Leiden, Niederlande), Glukose und LDH Veränderungen während einer $24 \mathrm{~h}$ Periode und anhand der Kontraktions bzw. Dilatations charakteristika der Arterien beurteilt.

\section{ERGEBNISSE:}

Vitalitätstests der Pilotstudien haben gezeigt, daß das arterielle Gewebe für z.Zt. 72 Stunden metabolisch unterstützt werden kann. So konnten Kontraktionen von bis zu $10 \%$ nach Verabreichung eines Norepinephrine Bolus beobachtet werden, was darauf hindeutet, daß die glatten Muskelzellen zumindest teilweise ihre Funktion beibehalten konnten. Darüberhinaus konnten spontane Durchmesseroszillationen beobachtet werden, die qualitativ gut mit in-vivo Studien übereinstimmen. Die Live/Dead ${ }^{\mathrm{TM}}$ Kollorierung der endothealen Zellkerne deuteten auf die Integrität des Endothels hin. Jedoch werden erst weitere Untersuchungen diese vorzeitigen Ergebnisse näher quantifizieren.

\section{DISKUSSION:}

Es ist die Überzeugung der Autoren, daß eine derartige Technologie ein vielversprechendes Potential in der Arterioskleroseforschung, in der Erforschung des Wall Remodeling und in der Überbrückung von Wartezeiten bei Organtransplantationen hat.

\section{LITERATUR:}

[1] Fry, D.L. et al., Arteriosclerosis and Thrombosis, Vol.12, p.357-368 (1992)

[2] Waliszewski et al., Proceedings of the 1995 Bioengineering Conference, BED-vol. 29, p.183184, 1995. 
